\title{
The Ionic Charge State Composition at High Energies in Large Solar Energetic Particle Events in Solar Cycle 23
}

\author{
R. A. Leske*, R. A. Mewaldt*, A. C. Cummings*, E. C. Stone* and \\ T. T. von Rosenvinge ${ }^{\dagger}$ \\ ${ }^{*}$ California Institute of Technology, Pasadena, CA 91125 USA \\ ${ }^{\dagger}$ NASA/Goddard Space Flight Center, Greenbelt, MD 20771 USA
}

\begin{abstract}
The ionic charge states of solar energetic particles (SEPs) depend upon the temperature of the source material and on the environment encountered during acceleration and transport during which electron stripping may occur. Measurements of SEP charge states at relatively high energies ( $\gtrsim 15 \mathrm{MeV} /$ nucleon) are possible with the Mass Spectrometer Telescope (MAST) on the Solar, Anomalous, and Magnetospheric Particle Explorer satellite by using the Earth's magnetic field as a particle rigidity filter. Using MAST data, we have determined ionic charge states of Fe and other elements in several of the largest SEP events of solar cycle 23. The charge states appear to be correlated with elemental abundances, with high charge states $(\sim 20 \mathrm{for} F e)$ for all elements in large Fe-rich events. We review the geomagnetic filter technique and summarize the results from MAST to date, with particular emphasis on new measurements in the very large 14 July 2000 SEP event. We compare the charge states determined by MAST with other measurements and with those expected from equilibrium calculations.
\end{abstract}

\section{INTRODUCTION}

Solar energetic particle (SEP) events are usually classified into one of two distinct types, gradual and impulsive [1]. Earlier studies found ionic charge states, $Q$, in gradual events to be similar to those expected for a plasma of $\mathrm{T}_{e} \sim 2 \mathrm{MK}$, with $Q(\mathrm{Fe}) \sim 15$ at $\sim 1 \mathrm{MeV} /$ nucleon and with little event-to-event variability [2]. Such temperatures are typical of the corona, lending support to the idea that particles in gradual SEP events originate as solar wind or coronal material accelerated by large shocks driven by coronal mass ejections. In impulsive events, however, significantly higher charge states of $\sim 20$ were found for $\mathrm{Fe}$ [3], suggesting an origin in hotter, $\sim 10^{7}$ $\mathrm{K}$ flare plasma or indicating that considerable stripping took place. Recent measurements from the Solar Energetic Particle Ionic Charge Analyzer (SEPICA) on the Advanced Composition Explorer (ACE) have revealed a broad continuum of charge states, varying from event to event but correlated with elemental abundances [4], suggesting a greater complexity than earlier measurements indicated. Also, acceleration or transport produces large elemental and isotopic fractionation in SEP events $[5,6]$ and $Q$ may also be affected.

Previous studies of ionic charge states using instruments employing electrostatic deflection [e.g. 2,7] were limited to $\sim 1 \mathrm{MeV} /$ nucleon. In recent years, these stud- ies have been extended to higher energies by using other techniques. One approach makes use of the Earth's magnetic field as a particle rigidity filter and has been successfully demonstrated at energies as high as $\sim 70$ $\mathrm{MeV} /$ nucleon [8] using the polar-orbiting Solar, Anomalous, and Magnetospheric Particle Explorer (SAMPEX) satellite. This same approach can be used at lower energies $[9,10,11]$, which allows for cross-calibration with direct measurements [7]. In addition to event-to-event variability, these measurements have shown that $Q$ may depend on energy $[10,11]$, which was confirmed by direct measurements at lower energies [7].

In this report, we illustrate the geomagnetic filter technique using data collected during the 14 July 2000 ("Bastille Day") SEP event by the Mass Spectrometer Telescope (MAST) on SAMPEX. We compare the new Bastille Day measurements with other SEP charge state measurements found by MAST to date and with charge states obtained by other methods. Further details of the analysis approach may be found in $[8,9,10,11,12]$.

\section{OBSERVATIONS}

SAMPEX was launched in July 1992 into a $520 \times 670 \mathrm{~km}$ $82^{\circ}$ inclination Earth orbit [13]. MAST employs a silicon solid-state detector telescope with a collecting power of 
$\sim 11 \mathrm{~cm}^{2}$ sr and uses the $\mathrm{d} E / \mathrm{d} x$ versus residual energy technique to measure the nuclear charge, $Z$, mass, $M$, and total kinetic energy, $E$, for particles with $E$ of $\sim 10$ to $\sim 100 \mathrm{MeV} /$ nucleon [14]. The energy interval depends on $Z$ and covers higher $E$ at higher $Z$, as illustrated in Figure 1 using data from the Bastille Day SEP event. In spite of the very high particle fluxes during this large event, all elements up through $\mathrm{Ni}(Z=28)$ are clearly resolved. MAST is not directly sensitive to the ionic charge of the particles, as incident ions at these energies are quickly stripped in traversing a few microns of material.

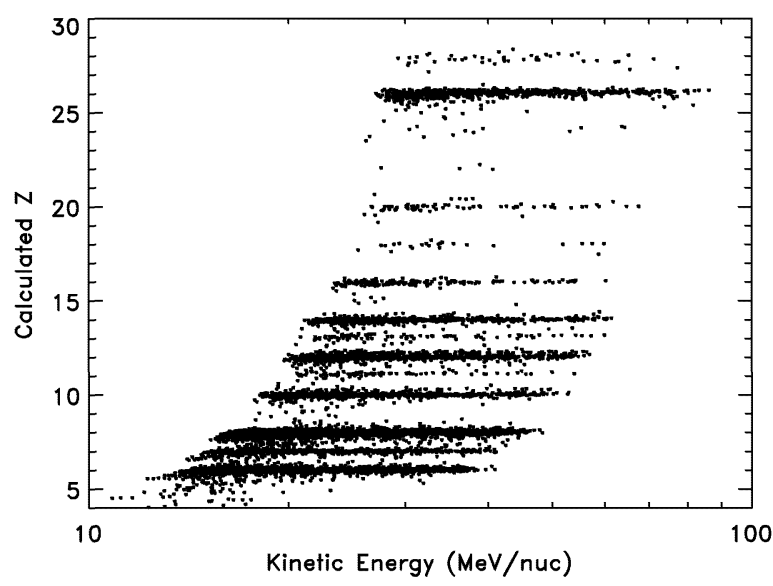

FIGURE 1. The calculated nuclear charge, $Z$, plotted versus kinetic energy for each heavy ion detected by SAMPEX/MAST from 14-21 July 2000 (DOY 196.0-203.0).

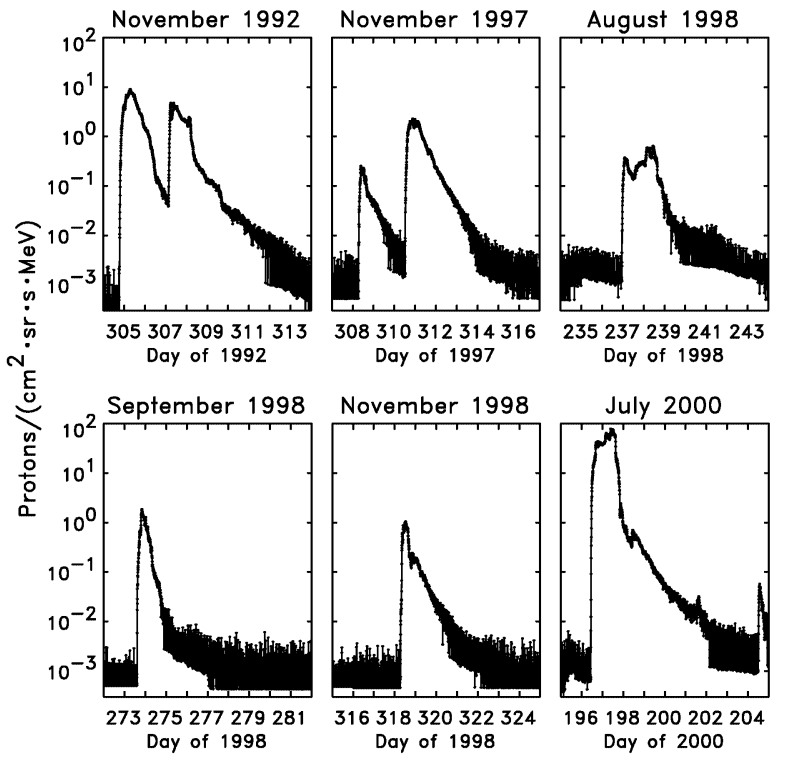

FIGURE 2. Time profiles of the SEP event periods examined here, using 5-min averaged intensities of $30-100 \mathrm{MeV}$ protons from GOES 7 and 8.

Time profiles of the events examined in this study are shown in Figure 2, using 30-100 $\mathrm{MeV}$ protons from the
GOES 7 and 8 spacecraft. The 1992 events occurred during the declining phase of solar cycle 22 , while the remainder of the events shown were in solar cycle 23. The Bastille Day event was the largest SEP event so far in solar cycle 23, with particle intensities up to two orders of magnitude greater than in some of the other events in this study. Of the events shown in Figure 2, the 4 November 1997 event is excluded from this study because of unfavorable spacecraft pointing conditions.

Heavy ions at high energies often arrive earlier and disappear sooner than protons in SEP events. In the Bastille Day event the shortest time profiles were found for the heaviest ions, as shown in Figure 3. The heavy ions peaked near the very beginning of the event and had practically vanished at these energies by late on 15 July. For the charge state analysis we integrated over days 196.5 to 198.0 of 2000 and have not subdivided this interval to search for time variations in $Q$.

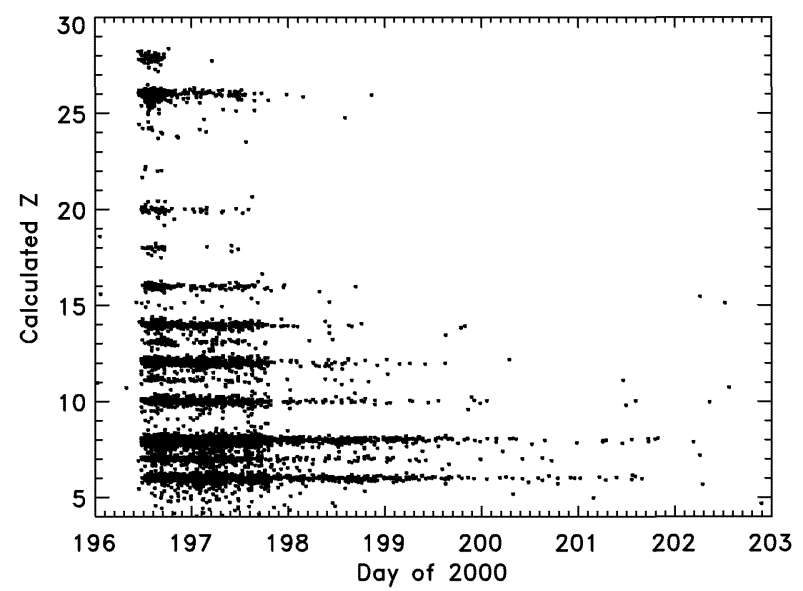

FIGURE 3. The calculated nuclear charge, $Z$, plotted versus arrival time for each heavy ion detected by SAMPEX/MAST during the Bastille Day event, illustrating the shorter time profiles for the heavier species.

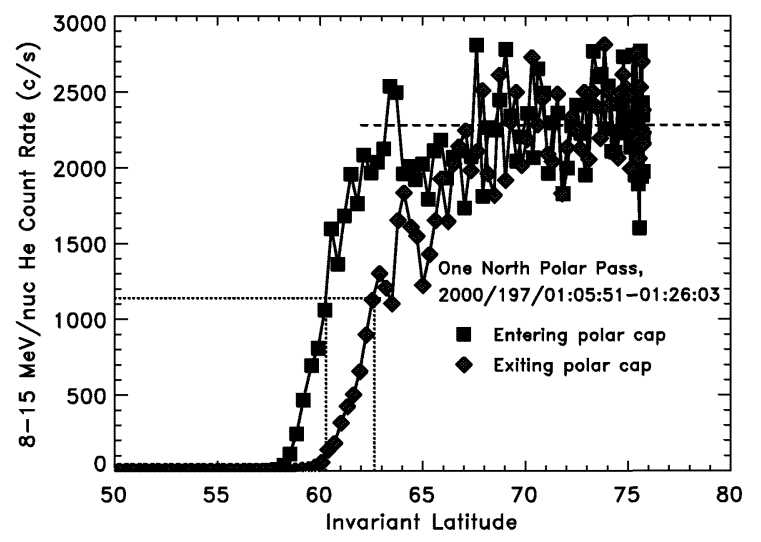

FIGURE 4. The count rate of 8-15 MeV/nucleon He measured every $6 \mathrm{~s}$ by MAST plotted versus invariant latitude for one crossing of the north polar cap on 15 July 2000. 


\section{ANALYSIS}

From the near-polar Earth orbit of SAMPEX, MAST samples energetic particles at all geomagnetic latitudes, including both the polar latitudes where SEPs can reach low altitudes and the equatorial regions where they are excluded by the geomagnetic field. The transition between these two regimes is evident in Figure 4, which shows the count rate for 8-15 MeV/nucleon He plotted versus invariant latitude, $\Lambda$, for a single passage over the north polar cap. (For any point along a magnetic field line, $\Lambda$ is the magnetic latitude at which the field line intersects the Earth's surface and is related to the magnetic $L$ shell by $\cos ^{2} \Lambda=1 / L$; see, e.g., [15]). We define the cutoff invariant latitude, $\Lambda_{C}$, to be that $\Lambda$ at which the count rate drops to half its average value above $70^{\circ}$, as indicated by dotted lines in Figure 4 . Since $\Lambda_{C}$ depends on the cutoff rigidity (momentum per unit charge), $R_{C}$, of the type of particle being measured, it is sensitive to the particle's charge state. By measuring $\Lambda_{C}$ for a given species and using the measurements of $E$ from MAST, one can solve for $Q$ if the relation between $\Lambda_{C}$ and $R_{C}$ is known. Calculations of $\Lambda_{C}$ differ from measured values by up to a couple of degrees even in geomagnetically quiet periods [16], and the dynamic nature of the cutoff during the geomagnetic disturbances which often accompany SEP events is even more difficult to model accurately [17]. However, we have found it possible to empirically determine both the time variations in the cutoff and the relation between $\Lambda_{C}$ and $R_{C}$.

When count rates are high, as in Figure 4, it is straightforward to measure $\Lambda_{C}$ up to 4 times every orbit, at each crossing into and out of both the north and south polar caps. This allows us to directly determine how the cutoff varies on relatively fine timescales even during severe geomagnetic storms. The orbit-averaged $\Lambda_{C}$ using the MAST 8-15 MeV/nucleon He rate during the Bastille Day event is shown in Figure 5. Cutoff variations in the earlier events and their relation to geomagnetic activity and local time (which accounts for the $\sim 2^{\circ}$ difference in $\Lambda_{C}$ from one side of the polar cap to the other in Figure 4) are discussed by Leske et al. [18]. The large cutoff suppression of $\gtrsim 10^{\circ}$ during the Bastille Day event is greater than any of the others studied with MAST [18] and corresponds to a severe geomagnetic storm when the geomagnetic activity index, Dst, reached $-300 \mathrm{nT}$.

The dependence of $\Lambda_{C}$ on species and energy is needed to obtain the ionic charge states. To determine the cutoffs for various $Z$ and $E$ with sufficient statistical accuracy, we must sum over the duration of the entire SEP event. Since the time profiles differ for each $Z$ and $E$ (Figure 3), we must first correct for the time variability of the cutoff, otherwise the cutoffs for different species would be weighted to different times, and temporal variations in $\Lambda_{C}$ would mask the dependence on $Z$ and $E$ we seek to

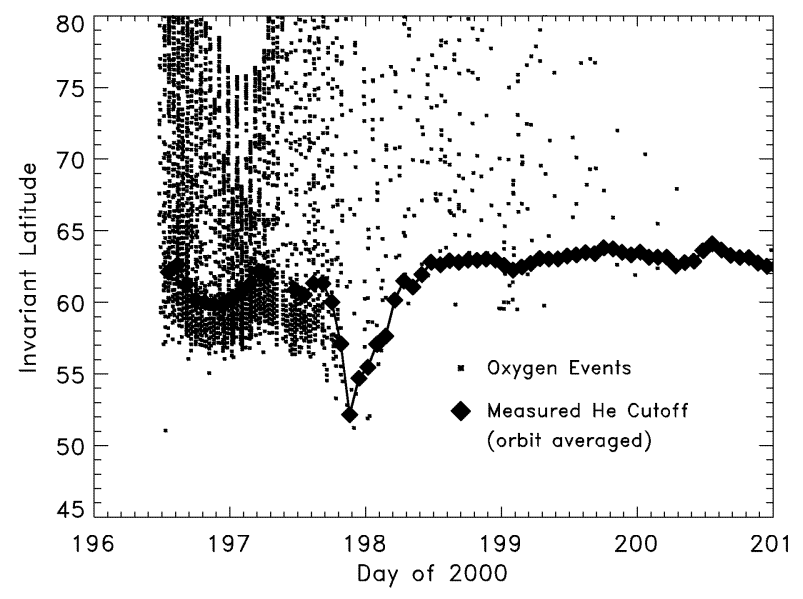

FIGURE 5. The invariant latitude at which each oxygen ion above $\sim 16 \mathrm{MeV} /$ nucleon was detected by MAST during the Bastille Day event, plotted versus time, compared with the time dependence of the orbit-averaged cutoff latitude determined from the 8-15 MeV/nucleon He rate.

measure. In the Bastille Day event, the particle intensity is low during the extreme cutoff suppression, so small errors in the time variability correction are less important. In other cases, however, especially those where two SEP events occur back-to-back as in November 1992 and November 1997 (Figure 2), the shock associated with the first event arrives near the peak of the second [18], and correcting for time variability is critical.

The invariant latitude at which each oxygen ion was detected by MAST during the Bastille Day event is also shown in Figure 5, and the abrupt lack of particles below $\sim 55^{\circ}$ is due to the geomagnetic cutoff. The cutoff is several degrees lower for the higher energy (and higher rigidity) oxygen, but the variability in the oxygen cutoff follows that determined from the He rate rather well. Therefore, we take $\Lambda_{C}$ measured using the rate of an abundant species such as $\mathrm{He}$ as a template to remove the cutoff variability from the higher $Z$ particles where more limited statistics prevents our measuring $\Lambda_{C}$ as accurately. For each detected particle, we find the difference between $\Lambda_{C}$ for He at the nearest cutoff crossing and the average He cutoff value, and subtract this difference from the $\Lambda$ at which the particle was detected.

After adjusting for the time variability, correcting for instrument livetime effects (which, for example, are responsible for the significant decrease in oxygen particles above the He cutoff in Figure 5), normalizing for the amount of time spent at each $\Lambda$, and summing over the duration of the event, the distributions in $\Lambda$ for the heavy elements resemble the rate profile shown in Figure $4[8,12]$. From linear fits to the low latitude edges of the distributions, we determine $\Lambda_{C}$ for each abundant species in several energy intervals. 
To derive a cutoff-rigidity relation, we assume that He is fully stripped $(Q=2)$ and that $Q(\mathrm{C})$ is between +5.7 (the average value found in gradual events at much lower energies [2]) and +6 (fully stripped). Calculations indicate that $R_{C}$ is linearly related to $\cos ^{4} \Lambda_{C}$ if $\Lambda_{C}$ is the vertical cutoff [e.g., 17]. As shown in Figure 6 (and as was clearer in the 1992 events $[8,12]$ ) our measurements agree with a linear relation. (It should also be noted that cutoff measurements using fully stripped nuclei find a linear relation from $\sim 500-1600 \mathrm{MV}$ during solar and geomagnetic quiet times [16]). A mean $Q$ for oxygen of $+7[2,8]$ agrees well with the trend established by $\mathrm{He}$ and $\mathrm{C}$ for both energies shown in Figure 6. Extrapolating the trend to higher rigidities, it is clear from the figure that $Q(\mathrm{Fe})$ must be $\sim 15$ or less; it seems very unlikely that $Q(\mathrm{Fe})$ could be, say, 20 or higher in this particular event. Linear fits to the combinations of the $\mathrm{He}$ and $\mathrm{C}$ data shown in the figure are used to establish the empirically-derived cutoff-rigidity relation for this event. Each $\Lambda_{C}$ measured for the various $Z$ and $E$ is converted to a rigidity using each of the three relations, and the resulting uncertainty in the average rigidity encompasses the full spread in values obtained from the three different relations. From the deduced $R_{C}$ and the measured $E$, the mean charge state and its uncertainty is obtained.

\section{RESULTS}

Charge states obtained by MAST in all of the SEP events studied to date, with their $1 \sigma$ uncertainties, are shown in Figure 7. Uncertainties in the derived cutoff-rigidity relations dominate the overall uncertainties and might be reduced through further analysis. Except for a slightly low value for $Q(\mathrm{Si})$, the charge states for the Bastille Day event are similar to those of the well-studied 1992 events.

A variety of other recent measurements of $Q$ in gradual events obtained using other techniques (mostly at similar energies) are also shown Figure 7. Charge states from ACE/SIS [19] were deduced assuming the elemental and isotopic fractionation observed in the 6 November 1997 SEP event both scale as the same power law in $Q / M$. While the relatively high $Q(\mathrm{Fe})$ value obtained from ACE/SIS agrees well with the value found by MAST in the same event, the Si charge states are very different. Other SAMPEX measurements in this event find $Q(\mathrm{Si}) \sim 13$ at $\sim 2.5 \mathrm{MeV} /$ nucleon and increasing with energy up to that point [11], apparently closer to the MAST value than to the value of $11.7 \pm 0.2$ deduced by Cohen et al. [19] at 12-60 MeV/nucleon. This may indicate systematic differences between the two approaches that are unaccounted for or additional stripping of $\mathrm{Si}$ after elemental and isotopic fractionation.

Values from the large gradual event of 20 April 1998

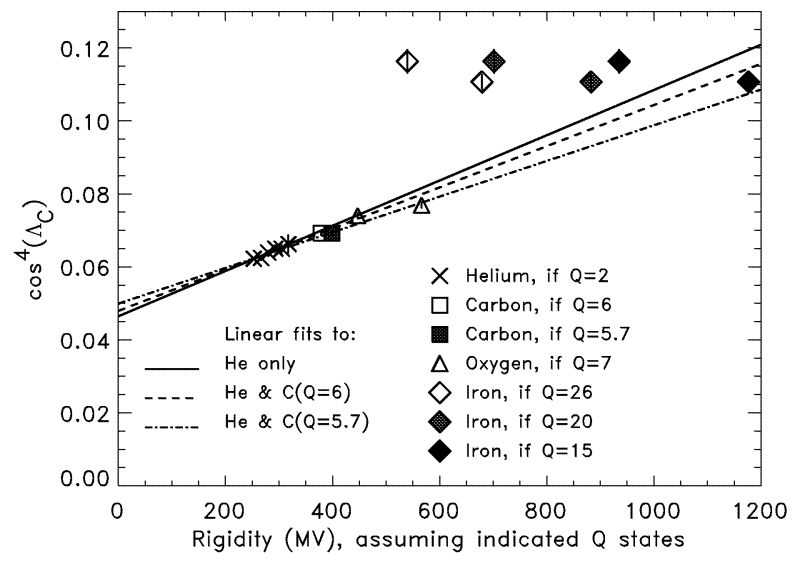

FIGURE 6. Measured $\cos ^{4} \Lambda_{C}$ during the Bastille Day event plotted versus rigidity calculated from the measured energy assuming the charge states indicated. For $\mathrm{O}$ and $\mathrm{Fe}$, data at 2 energy points (at 3 different trial charge states each for $\mathrm{Fe}$ ) are shown. Lines are fits to the subsets of the data indicated and serve as the relations between $\Lambda_{C}$ and $R_{C}$ used to obtain $Q$.

(not studied by MAST) shown from the work of Tylka et al. [24] were obtained from fits to the elemental energy spectra over a broad energy interval and agree well with the direct measurements from ACE/SEPICA [23] at much lower energies. For most elements, the measured charge states are often close to those expected from equilibrium calculations of collisional ionization in a 2 MK plasma [21, 22], a temperature typical of the solar corona. For the MAST 1997 and 1998 events shown, however, the high charge states of $\sim 20$ for Fe either require a higher temperature of $\sim 10 \mathrm{MK}$ or may suggest that additional stripping occurs during acceleration or transport [25, 26]. High energy tails on nonMaxwellian electron distributions might also generate somewhat higher charge states even in an otherwise relatively cold plasma, but this seems to be a larger effect for ions with a higher ionization potential such as $\mathrm{O}$ rather than $\mathrm{Fe}$ [e.g., 27, 28].

At lower energies of $\sim 1 \mathrm{MeV} /$ nucleon, a good correlation has been reported between $Q(\mathrm{Fe})$ and the $\mathrm{Fe} / \mathrm{O}$ ratio, with higher $Q$ corresponding to enhanced $\mathrm{Fe} / \mathrm{O}$ [4]. A similar trend is evident here, as shown in Table 1 using MAST $Q$ measurements and the $\mathrm{Fe} / \mathrm{O}$ ratio (for most of the events) obtained by ACE/SIS [5]. For the 25 August 1998 event, the $\mathrm{Fe}$ abundance above $30 \mathrm{MeV} /$ nucleon was so low that it was not possible to accurately measure the cutoff location with the few particles detected by MAST, so no value for $Q$ is given. Comparing Table 1 with Figure 2 of [4], it appears that for a given $\mathrm{Fe} / \mathrm{O}$ value, $Q(\mathrm{Fe})$ at MAST energies is generally higher than the value at lower energies. This may suggest that an increase in $Q(\mathrm{Fe})$ with energy is fairly common, as has been measured in at least two events $[7,10,11]$. 


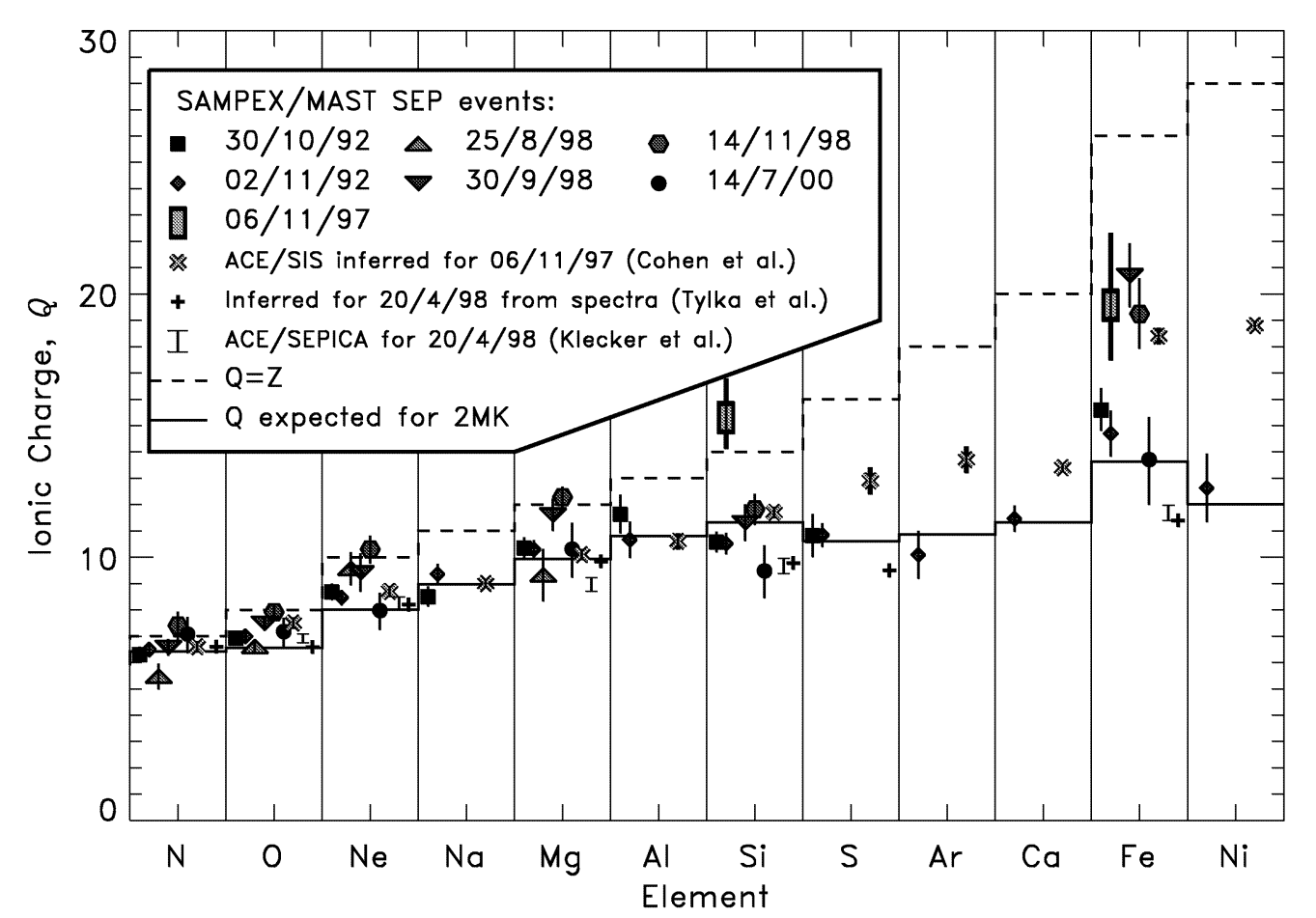

FIGURE 7. Charge states determined by MAST in SEP events in 1992 [8, 12], 1997 [11], 1998 [20], and 2000 (this work) compared with those expected in a 2 MK plasma (solid horizontal lines) [21, 22]. For comparison, charge states deduced in the 6 November 1997 event at similar energies are also shown [19], as well as charge states directly measured at low energies [23] and deduced from the spectral shape at high energies [24] in the 20 April 1998 event.

TABLE 1. $\mathrm{Fe} / \mathrm{O}$ and $Q(\mathrm{Fe})$ (at $E \sim 28-65 \mathrm{MeV} /$ nucleon) for MAST SEP events.

\begin{tabular}{ccc}
\hline Event & Fe/O & $Q(\mathbf{F e})$ \\
\hline $30 / 10 / 92$ & $0.0702 \pm 0.0096^{*}[29]$ & $15.59 \pm 0.81[8]$ \\
$02 / 11 / 92$ & $0.0932 \pm 0.0054^{*}[29]$ & $14.69 \pm 0.86[8]$ \\
$06 / 11 / 97$ & $0.900 \pm 0.006^{\dagger}[5]$ & $19.6 \pm 2.4[11]$ \\
$25 / 08 / 98$ & $0.016 \pm 0.001^{\dagger}[5]$ & N/A \\
$30 / 09 / 98$ & $0.299 \pm 0.003^{\dagger}[5]$ & $20.7 \pm 1.2[20]$ \\
$14 / 11 / 98$ & $0.761 \pm 0.006^{\dagger}[5]$ & $19.2 \pm 1.3[20]$ \\
$14 / 07 / 00$ & $0.0377 \pm 0.0007^{\dagger * *}$ & $13.7 \pm 1.7^{\ddagger}$ \\
\hline
\end{tabular}

${ }^{*} E=30-60 \mathrm{MeV} /$ nucleon

${ }^{\dagger} E=12-60 \mathrm{MeV} /$ nucleon

** From ACE/SIS [C. M. S. Cohen, private communication]

$¥$ This work

It is unclear whether the correlation of composition and charge states is due to an admixture of impulsive SEP material into these otherwise gradual events [30], an origin of the material in hotter regions of the corona, or fractionation or charge-changing processes during acceleration or transport. The $Q / M$-dependent fractionation process that affects both elemental and isotopic abundances [e.g., 6] would not produce this correlation from material with a broad distribution of $\mathrm{Fe}$ charge states. If it did, then when $\mathrm{Fe} / \mathrm{O}$ is enhanced, indicating enhancements of
TABLE 2. Mean $Q$ for Fe-poor and Fe-rich gradual events from MAST.

\begin{tabular}{ccc}
\hline Element & avg $Q$, low $\mathbf{~ F e} / \mathbf{O}^{*}$ & avg $Q$, high $\mathbf{~ F e} / \mathbf{O}^{\dagger}$ \\
\hline $\mathrm{N}$ & $6.36 \pm 0.15$ & $6.79 \pm 0.27$ \\
$\mathrm{O}$ & $6.90 \pm 0.13$ & $7.68 \pm 0.21$ \\
$\mathrm{Ne}$ & $8.63 \pm 0.19$ & $9.99 \pm 0.42$ \\
$\mathrm{Mg}$ & $10.26 \pm 0.24$ & $12.07 \pm 0.33$ \\
$\mathrm{Si}$ & $10.47 \pm 0.27$ & $(11.98 \pm 0.42)^{* *}$ \\
$\mathrm{Fe}$ & $15.02 \pm 0.56$ & $19.97 \pm 0.82$ \\
\hline
\end{tabular}

* Average of events with $\mathrm{Fe} / \mathrm{O}<0.134$

$\dagger$ Average of events with $\mathrm{Fe} / \mathrm{O}>0.134$

** Average omitting the $06 / 11 / 97$ event is $11.61 \pm 0.44$

elements with lower $Q / M$, the lower $Q(\mathrm{Fe})$ should also be enhanced, opposite to what is observed.

Using Table 1 , if we divide the data into events where the high energy $\mathrm{Fe} / \mathrm{O}$ ratio is depleted relative to the SEP-derived coronal value of 0.134 [31] or enhanced relative to the corona and calculate the average $Q$ for each element in each of these two cases, we obtain the values given in Table 2 . Because $Q(\mathrm{Si})$ in the 6 November 1997 event is unusually high compared to that in the other two Fe-rich events, we list values in Table 2 both with and without this event included in the average. Both values are significantly higher than the average $Q(\mathrm{Si})$ in 
the Fe-poor events. In fact, for all 6 measured elements from $\mathrm{N}$ to $\mathrm{Fe}$, the mean $Q$ is significantly higher for the Fe-rich events, being some 5 charge units higher at $\mathrm{Fe}$ and essentially consistent with fully stripped for all elements up through Mg. Table 2 agrees with lower energy observations showing charge states for different elements tend to track each other [4], and the $Q$ values are very similar to those deduced indirectly by ACE/SIS in $4 \mathrm{Fe}$-rich gradual events [5].

Measurements from SAMPEX have uncovered unexpected behavior in gradual SEP event charge states. The analysis of additional large events detected by MAST, namely the 9 November 2000 event and several large events in March and April of 2001, is underway. Together with ongoing composition measurements from ACE, Wind, and other spacecraft, these new data offer the hope of revealing new details of the fractionation, acceleration, and transport of solar energetic particles.

\section{ACKNOWLEDGMENTS}

This research was supported by NASA under contract NAS5-30704 and grant NAGW-1919. GOES data (Figure 2) were obtained from the National Oceanic and Atmospheric Administration (NOAA), US Department of Commerce, through the Space Environment Center and the National Geophysical Data Center.

\section{REFERENCES}

1. Reames, D. V., Revs. Geophys., 33, 585-589 (1995).

2. Luhn, A., et al., Proc. 19th Internat. Cosmic Ray Conf. (La Jolla), 4, 241-244 (1985).

3. Luhn, A., Klecker, B., Hovestadt, D., and Möbius, E., Astrophys. J., 317, 951-955 (1987).

4. Möbius, E., et al., "Survey of Ionic Charge States of Solar Energetic Particle Events During the First Year of ACE", in Acceleration and Transport of Energetic Particles Observed in the Heliosphere: ACE 2000 Symposium, edited by R. A. Mewaldt et al., AIP Conf. Proc. 528, AIP, New York, 2000, pp. 131-134.

5. Cohen, C. M. S., et al., Geophys. Res. Lett., 26, 2697-2700 (1999).

6. Leske, R. A., et al., "Isotopic Abundances in the Solar Corona as Inferred from ACE Measurements of Solar Energetic Particles", in Proc. Joint SOHO-ACE Workshop 2001, AIP Conf. Proc., AIP, New York, 2001, this volume.

7. Möbius, E., et al., Geophys. Res. Lett., 26, 145-148 (1999).

8. Leske, R. A., Cummings, J. R., Mewaldt, R. A., Stone, E. C., and von Rosenvinge, T. T., Astrophys. J. Lett., 452, L149-L152 (1995).

9. Mason, G. M., Mazur, J. E., Looper, M. D., and Mewaldt, R. A., Astrophys. J., 452, 901-911 (1995).

10. Oetliker, M., et al., Astrophys. J., 477, 495-501 (1997).
11. Mazur, J. E., Mason, G. M., Looper, M. D., Leske, R. A., and Mewaldt, R. A., Geophys. Res. Lett., 26, 173-176 (1999).

12. Leske, R. A., Cummings, J. R., Mewaldt, R. A., Stone, E. C., and von Rosenvinge, T. T., "Measurements of the Ionic Charge States of Solar Energetic Particles at 15-70 MeV/nucleon Using the Geomagnetic Field", in High Energy Solar Physics, edited by R. Ramaty, N. Mandzhavidze, and X.-M. Hua, AIP Conf. Proc. 374, AIP, New York, 1996, pp. 86-95.

13. Baker, D. N., et al., IEEE Trans. Geosci. Remote Sensing, 31, 531-541 (1993).

14. Cook, W. R., et al., IEEE Trans. Geosci. Remote Sensing, 31, 557-564 (1993).

15. Roederer, J. G., Dynamics of Geomagnetically Trapped Radiation, Springer-Verlag, New York, 1970.

16. Ogliore, R. C., Mewaldt, R. A., Leske, R. A., Stone, E. C., and von Rosenvinge, T. T., Proc. 27th Internat. Cosmic Ray Conf. (Hamburg) (2001), in press.

17. Smart, D. F., Shea, M. A., Flückiger, E. O., Tylka, A. J., and Boberg, P. R., Proc. 26th Internat. Cosmic Ray Conf. (Salt Lake City), 7, 337-340 (1999).

18. Leske, R. A., Mewaldt, R. A., Stone, E. C., and von Rosenvinge, T. T., J. Geophys. Res. (2001), in press.

19. Cohen, C. M. S., et al., Geophys. Res. Lett., 26, 149-152 (1999).

20. Larson, D. J., et al., Proc. 26th Internat. Cosmic Ray Conf. (Salt Lake City), 7, 301-304 (1999).

21. Arnaud, M., and Rothenflug, R., Astron. Astrophys. Suppl., 60, 425-457 (1985).

22. Arnaud, M., and Raymond, J., Astrophys. J., 398, 394-406 (1992).

23. Klecker, B., et al., Proc. 26th Internat. Cosmic Ray Conf. (Salt Lake City), 6, 83-86 (1999).

24. Tylka, A. J., Boberg, P. R., McGuire, R. E., Ng, C. K., and Reames, D. V., "Temporal Evolution in the Spectra of Gradual Solar Energetic Particle Events", in Acceleration and Transport of Energetic Particles Observed in the Heliosphere: ACE 2000 Symposium, edited by R. A. Mewaldt et al., AIP Conf. Proc. 528, AIP, New York, 2000, pp. 147-152.

25. Barghouty, A. F., and Mewaldt, R. A., "Simulation of Charge-Equilibration and Acceleration of Solar Energetic Ions", in Acceleration and Transport of Energetic Particles Observed in the Heliosphere: ACE 2000 Symposium, edited by R. A. Mewaldt et al., AIP Conf. Proc. 528, AIP, New York, 2000, pp. 71-78.

26. Reames, D. V., Ng, C. K., and Tylka, A. J., Geophys. Res. Lett., 26, 3585-3588 (1999).

27. Owocki, S. P., and Scudder, J. D., Astrophys. J., 270, 758-768 (1983).

28. Ko, Y.-K., Fisk, L. A., Gloeckler, G., and Geiss, J., Geophys. Res. Lett., 23, 2785-2788 (1996).

29. Williams, D. L., Measurements of the Isotopic Composition of Solar Energetic Particles with the MAST Instrument Aboard the SAMPEX Spacecraft, Ph.D. thesis, California Institute of Technology, Pasadena, CA 91125, USA (1998).

30. Mason, G. M., Mazur, J. E., and Dwyer, J. E., Astrophys. J. Lett., 525, L133-L136 (1999).

31. Reames, D. V., Adv. Space Res., 15, (7)41-(7)51 (1995). 